\title{
Urge Urinary Incontinence. Surgical Treatment
}

\author{
Mateo Hevia, José Enrique Robles
}

Department of Urology, Clínica Universidad de Navarra, Pamplona, Spain

\begin{abstract}
Urge urinary incontinence (UUI) is defined as the involuntary loss of urine preceded by mictional imperiousness or inability to delay micturition. These losses may vary in grade and frequency, with the consequent impact on the quality of life. The pathogenesis has not been resolved satisfactorily, existing nowadays various theories about the etiology of this entity related overall to the innervation of the detrusor muscle and the sensitivity of the bladder mucosa.

UUI is a very common problem among the female population that significantly affects the quality of life. The exact prevalence cannot be known due to the tendency of many women to hide the problem. According to studies, the percentage of UUI varies between 10 and $33 \%$ of women with incontinence (any involuntary loss of urine).
\end{abstract}

Unlike stress urinary incontinence (SUI), where the effectiveness of surgical treatment is well known, for UUI the available and widespread therapeutic option has been until recent time to try to improve symptoms with medication (anticholinergics, beta-3 agonists) or other procedures (botulinum toxin), only thinking about surgical treatment at the cases with extremely poor outcome (enterocystoplasty, cystectomy).

About 25 years ago, UUI began to be attributed to an anatomical defect of the pelvic floor (De Lancey, Petros). More recently, Jäger W. figured out that the increased urinary frequency of the studied women was only during daytime, i.e., being symptom-free in repose. In addition, women with pelvic organ prolapse and urinary urgency referred improvement or disappearance of symptoms after prolapse surgical repair. Therefore, it could be concluded that UUI is caused in a significant percentage of cases by a position-dependent phenomenon, essentially by the base-neck of the bladder.

Based on this theory, the treatment of such defect would be the solution for UUI and the other symptoms that are part of the overactive bladder syndrome (urinary urgency and frequency); being the position of the vaginal vault which determines the effectiveness of the treatment, due to the support function that it exerts on bladder neck.

Thus, Jäger developed a technique for the treatment of UUI which was also used for pelvic organ prolapse, called VA(gino)RE(cto)SA(cropexy) or CE(rvico)RE(cto)SA(cropexy) (if uterus present, where you can perform a supracervical hysterectomy during surgery).

In addition, based on the results from preliminary studies with corpses and experimental animals, Jäger started to work on the hypothesis that UUI could only be cured if the utero-sacral ligaments (USL) were replaced with bands, even in patients without clinically pelvic organ prolapses. Moreover, for the location to fix these bands, made of plastic polyvinylidene fluoride (PVDF), S2/S3 periosteum was decided as it is the anatomical origin of USL and due to the not achievement of the desired effect for UUI with promontorium (like sacrocopopexy). It was found out as well that it was mandatory to replace both USL, as in patients where one of the two bands was broken, UUI immediately recurred. There were no erosions as the location of both bands follow the natural way of USL.

Following the theory of Petros ("Intregal Theory"), both USL and pubo-urethral ligaments (PUL) have to be substituted to restore the pelvic floor function. Therefore, in addition to the performed procedure, either VA(RE)SA or CE(RE)SA, a suburethral TOT mesh was also placed. However, as TOT placement was delayed in time due to organizational issues, after 3 months UUI was resolved in up to $40 \%$ of patients without the need of PUL replacement (i.e., without TOT). It means that in $60 \%$ of cases a complete restoration of the pelvic floor with both PUL and USL was needed. 
Currently, a large number of patients treated with the different variations of the technique are being followed (nearly thousand) with a good percentage of initial healing, in order to compare them with the current standards of treatment.

With these advances in surgical treatment it seems obvious that we have a range of possibilities for facing UUI. 